\title{
Emergency Ultrasound Predicting the Need for Therapeutic Laparotomy among Blunt Abdominal Trauma Patients in a Sub-Saharan African Hospital
}

\author{
P. C. M. Musiitwa, ${ }^{1}$ M. Galukande, ${ }^{1}$ S. Bugeza, ${ }^{2}$ H. Wanzira, ${ }^{3}$ and R. Wangoda ${ }^{1}$ \\ ${ }^{1}$ Department of Surgery, College of Health Sciences, Makerere University, Kampala, Uganda \\ ${ }^{2}$ Department of Radiology, College of Health Sciences, Makerere University, Kampala, Uganda \\ ${ }^{3}$ Infectious Diseases Research Collaboration, Makerere University, Kampala, Uganda \\ Correspondence should be addressed to M. Galukande; mosesg@img.co.ug
}

Received 27 June 2013; Revised 19 December 2013; Accepted 3 January 2014; Published 13 February 2014

Academic Editor: Rade B. Vukmir

Copyright (C) 2014 P. C. M. Musiitwa et al. This is an open access article distributed under the Creative Commons Attribution License, which permits unrestricted use, distribution, and reproduction in any medium, provided the original work is properly cited.

Background. The trauma burden globally accounts for high levels of mortality and morbidity. Blunt abdominal trauma (BAT) contributes significantly to this burden. Patient's evaluation for BAT remains a diagnostic challenge for emergency physicians. SSORTT gives a score that can predict the need for laparotomy. The objective of this study was to assess the accuracy of SSORTT score in predicting the need for a therapeutic laparotomy after BAT. Method. A prospective observational study. Eligible patients were evaluated for shock and the presence of haemoperitoneum using a portable ultrasound machine. Further evaluation of patients following the standard of care (SOC) protocol was done. The accuracy of SSORTT score in predicting therapeutic laparotomy was compared to SOC. Results. In total, 195 patients were evaluated; $\mathrm{M}: \mathrm{F}$ ratio was $6: 1$. The commonest injuries were to the head 80 $(42 \%)$ and the abdomen 54 (28\%). A SSORTT score of $>2$ appropriately identified patients that needed a therapeutic laparotomy (with sensitivity 90\%, specificity 90\%, PPV 53\%, and NPV 98\%). The overall mortality rate was $17 \%$. Conclusion. Patients with a SSORTT score of 2 and above had a high likelihood of requiring a therapeutic laparotomy. SSORTT scoring should be adopted for routine practice in low technology settings.

\section{Introduction}

Trauma burden globally is on the increase accounting for high levels of mortality and morbidity. It is the second largest single cause of disease accounting for $16 \%$, only second to parasitic and infectious diseases at $23 \%$. WHO also estimates that, by the year 2020 , trauma will be the first or second leading cause of "years of productive life lost" for the entire world population in both developed and developing world [1].

Blunt abdominal trauma (BAT) is injury to intraabdominal or retroperitoneal viscera as a result of a nonpenetrating force. It is commonly due to rapid deceleration or acceleration in combination with shearing and rotational forces $[2,3]$. Common manifestations are hemorrhage and visceral perforation, either intraperitoneal or retroperitoneal; morbidity and mortality are associated with delay in diagnosis and treatment of BAT [4-6].

Common causes include road traffic crushes (RTC), industrial accidents, falls from heights, sporting, and farm accidents (in rural areas) and sometimes the cause of BAT is unknown, for example, with child abuse and domestic violence $[7,8]$.

Evaluation of patients with BAT is often a diagnostic challenge for emergency physicians and trauma surgeons $[9,10]$. Uncontrolled hemorrhage is responsible for over $50 \%$ of trauma related deaths [11-13].

Other times overly aggressive management can lead to nontherapeutic laparotomy. The negative laparotomy rate is $16 \%$ and negative laparotomy carries the risk of incisional 
hernia and small bowel obstruction over time. Emergent sonography in form of Focused Abdominal Sonography for Trauma (FAST) has emerged and been embraced as a rapid, noninvasive, and accurate method of evaluating blunt abdominal trauma that can be easily used by emergency room clinicians and trauma surgeons [14-17]. FAST is a rapid, fourview ultrasound examination carried out during the primary survey that assesses for haemoperitoneum, haemothorax, and haemopericardium.

A newer dimension of emergency sonography is the Sonographic Scoring for Operating Room Triage in Trauma (SSORTT score) which was developed in a trauma center in the United States of America with the aim of enabling emergency room clinicians to quickly and reliably predict which patients require an emergent laparotomy. It has three components: (1) FAST findings, (2) systolic blood pressure and, (3) pulse rate which together give a score that can predict whether patient would most likely not require a therapeutic laparotomy.

The objective of this study therefore was to determine whether specific SSORTT scores are good predictors of a need for a therapeutic laparotomy among blunt abdominal trauma patients in a low resource settings.

\section{Methods}

2.1. Study Design. A prospective observational cohort study carried out from December 2012 to April 2013.

2.2. Study Setting. The study was conducted in the 24/7 A and E department of Mulago National referral hospital situated in Kampala, Uganda. This is a fully fledged unit with a medical and surgical emergency wing, two operating rooms, an X-ray facility, ultrasound facility, resuscitation room with 3 beds, and a 26-bed holding emergency ward; adjacent to it are the blood bank, hematology, microbiology, and chemistry laboratories.

It receives about 1500 trauma patients per month, 300 with suspected torso injuries and 20 with actual torso injuries [18].

2.3. Study Procedure. Upon arrival in the A\&E unit patients with suspected BAT were screened.

BAT was defined as injury to intra-abdominal viscera or retroperitoneal as a result of a nonpenetrating object or force indicated by presence of torso abrasions and bruises, abdominal tenderness among multiply injured patients with long bone fractures, fracture pelvis, and spine injuries.

Patients meeting these eligibility criteria were recruited and subsequently informed written consent was obtained. All patients were adequately resuscitated and investigated as per the standard of care protocol. Patients with subcutaneous emphysema over the abdomen in whom abdominal assessment with sonography would be difficult to interpret were excluded.

Assessment was done using ATLS protocol. History and physical examination findings were recorded on pre-coded questionnaires. Patients suspected to be having BAT were positioned in supine position and underwent FAST using SonoSite TITAN portable ultrasound machine with a transducer frequency ranging from 3.5 to $5 \mathrm{MHz}$. A cumulative sum of the three parameters ultrasound score, systolic blood pressure, and pulse rate were determined and used as the SSORTT score.

Patient then underwent secondary survey, which was followed by other routine investigations and management according to the hospital guidelines. As and when appropriate patients were sent to the emergency radiology room for a full ultrasound scan and abdominal X-ray. All admitted patients were followed up for three days to determine whether they had laparotomy or not.

2.4. Study Variables. These included the SSORTT score, age, sex, and mechanism of injury, for example, RTC. In addition and therapeutic laparotomy, nontherapeutic laparotomy, and outcomes of nonoperative management (lived/died).

2.5. Data Collection, Management, and Analysis. Data were collected using pretested questionnaires and double entered, coded, and cleaned using Epi data version 5.3.2 software. Stored data were exported to Stata version 12 for analysis. Accuracy of the SSORTT score in predicting patients that would need therapeutic laparotomy was determined by calculating sensitivity, specificity, PPV, NPV, and ROC curve of SSORTT score. ROC curve was used to determine diagnostic accuracy of SSORTT score for predicting the need for therapeutic laparotomy.

2.6. Quality Control. The investigator(s) underwent didactic tutorials on FAST also performed at least 30 ultrasound scans to determine presence of haemoperitoneum prior to starting the study.

The ultrasound machine was maintained and calibrated by hospital staff from the department of radiology. We stored the examinations images (sonographs) and had them reread (validated) by a consultant radiologist.

2.7. Ethical Considerations. Written informed consent for patients above 18 years and assent for those less than 18 years were obtained from the participants. Ethical approval was obtained from the Research and Ethics Committee of the College of Health Sciences of Makerere University.

\section{Results}

A total of 195 trauma patients clinically suspected to have sustained BAT were subjected to emergency ultrasound scanning and were SSORTT scored at the A\&E unit of Mulago Hospital from December 2012 to April 2013. The study profile is shown in Figure 1. The patients' characteristics and laparotomy findings are outlined in Table 1.

The male: female ratio was $6: 1$. Age group between 20 and 40 years comprised majority of patients 153 (79\%). Road traffic crashes (RTC) were the leading cause of injury in this study accounting for $59 \%$.Others causes included assault $32 \%$, falls $7 \%$, and others $2 \%$ as shown in Table 1 . 


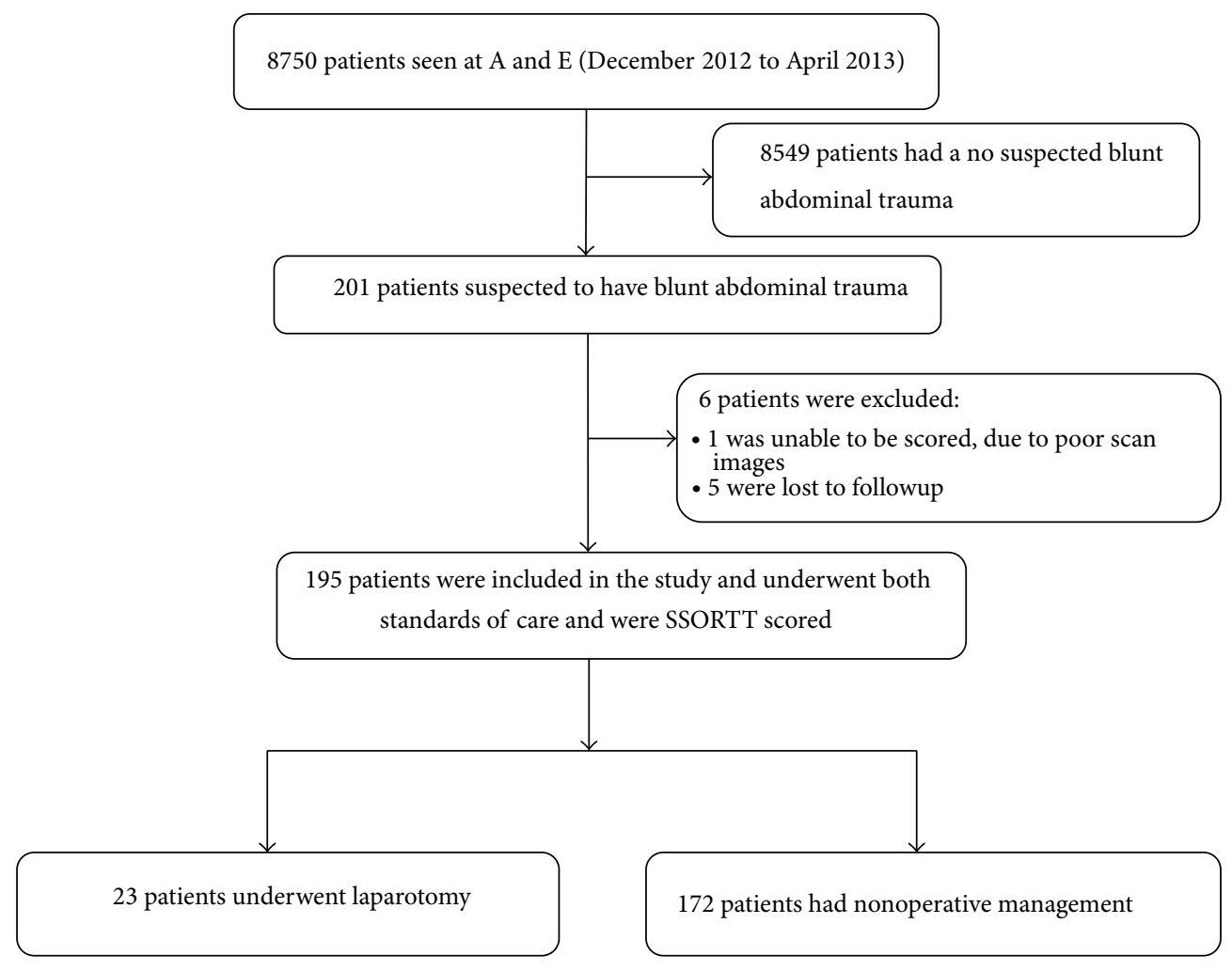

FIGURE 1: Study profile.

Most of patients with BAT had clinically suffered injuries to the head, abdomen, or limbs.

Table 1 shows the distribution of SSORTT scores by intervention. 20/23 (86\%) patients who had therapeutic laparotomy had a SSORTT score of $\geq 3.156 / 173$ (90\%); patients who had nonoperative management had a SSORTT score of $\leq 2$.

Table 2 shows the laparotomy predictions at selected cut offs.

Table 3 shows that the majority of patients who had a laparotomy had high Glasgow coma scores. A small proportion had Glasgow coma score $<13$.

More than $80 \%$ of the patients with abdominal tell-tale signs and head injury were subjected to a laparotomy.

Majority of the patients with head injury were subjected to nonoperative management.

Among therapeutic laparotomy patients, massive haemoperitoneum was the commonest finding (47\%), followed by solid organ injury at $35 \%$ and gut perforation at $6 \%$.

Among nontherapeutic laparotomy retroperitoneal hematoma and <grade3 solid organs injury were findings.

The general trend for sensitivity at the various cut-off levels of the SSORTT score was that it reduced with an increasing score; see Table 3.

Values of specificity gradually increase as

at SSORTT score 0 , sensitivity $=91 \%$ and specificity $=$ $82 \%$; at SSORTT score 6 , sensitivity $=0 \%$ and specificity $=$ $100 \%$;

at SSORTT score 0, PPV $=39 \%$ and $\mathrm{NPV}=99 \%$;

at SSORTT score $6, \mathrm{PPV}=0 \%$ and NPV $=89 \%$.

3.1. Accuracy . For Accuracy of the Test, the following ranking was considered:

$$
\begin{aligned}
& .90-1=\text { excellent }(\mathrm{A}) ; \\
& .80-.90=\text { good }(\mathrm{B}) ; \\
& .70-.80=\text { fair }(\mathrm{C}) ; \\
& .60-.70=\text { poor }(\mathrm{D}) ; \\
& .50-.60=\text { fail }(\mathrm{F}) .
\end{aligned}
$$

The area under the ROC curve measured accuracy; see Figure 2.

The closer the curve follows the left-hand border and then the top borders of the ROC space, the more accurate the test is. The area under ROC curve measured 0.91 a high value for accuracy.

The presence of signs which included bruises, abrasions, lacerations, and cuts did not predict staying alive or dying. The patients who had a laparotomy were less likely to die OR 1.64 though it was not statistically significant $P=0.370$.

Of 23 patients that had laparotomy 21 patients had therapeutic laparotomy and 2 being nontherapeutic, one had a moderately large retroperitoneal haematoma and the other 
TABLE 1: Distribution of baseline characteristics and laparotomy findings for the 195 participants.

\begin{tabular}{|c|c|c|}
\hline \multirow{2}{*}{ Characteristics } & \multicolumn{2}{|c|}{ Participants } \\
\hline & Number & Percentage \\
\hline \multicolumn{3}{|l|}{ Age groups } \\
\hline$<20$ & 22 & 11 \\
\hline $20-40$ & 153 & 79 \\
\hline $41-60$ & 18 & 9 \\
\hline$>60$ & 2 & 1 \\
\hline \multicolumn{3}{|l|}{ Gender } \\
\hline Male & 168 & 86 \\
\hline Female & 27 & 14 \\
\hline \multicolumn{3}{|l|}{ Time since injury in hours* } \\
\hline$<1$ & 20 & 10 \\
\hline $1-2$ & 84 & 43 \\
\hline$>2$ & 73 & 37 \\
\hline Unknown & 18 & 10 \\
\hline \multicolumn{3}{|l|}{ Mechanism of injury* } \\
\hline Road traffic crash & 116 & 59 \\
\hline Assault & 62 & 32 \\
\hline Fall & 13 & 6 \\
\hline Others & 4 & 2 \\
\hline \multicolumn{3}{|l|}{ Therapeutic laparotomy } \\
\hline Massive haemoperitoneum & 16 & 47 \\
\hline Grade $4 / 5$ solid organs injury & 12 & 35 \\
\hline Gut perforation & 2 & 6 \\
\hline Mesenteric tears & 1 & 3 \\
\hline Bladder injury & 1 & 3 \\
\hline \multicolumn{3}{|l|}{ Nontherapeutic laparotomy } \\
\hline Retroperitoneal haematoma & 1 & 3 \\
\hline Grade 3 solid organs injury & 1 & 3 \\
\hline \multirow[t]{2}{*}{ SSORTT score } & $\begin{array}{l}\text { Laparotomy } \\
\qquad N=23\end{array}$ & $\begin{array}{c}\text { No laparotomy } \\
\quad N=172\end{array}$ \\
\hline & Number (\%) & Number (\%) \\
\hline 0 & $2(9)$ & $142(83)$ \\
\hline 1 & 0 & $13(8)$ \\
\hline 2 & $1(4)$ & $1(1)$ \\
\hline 3 & $5(22)^{*}$ & $12(7)$ \\
\hline 4 & $6(26)$ & $3(2)$ \\
\hline 5 & $3(13)$ & $1(1)$ \\
\hline 6 & $6(26)^{*}$ & $1(0)$ \\
\hline
\end{tabular}

${ }^{*}$ Two participants had nontherapeutic laparotomy with scores of 3 and 6.

had a grade two splenic laceration with a haemoperitoneum of about $500 \mathrm{cc}$. Of the 172 patients that did not undergo laparotomy 28 patients passed away and the rest were either discharged or still admitted by day three. Of the 28 patients, 18 patients were multiply injured with severe extra-abdominal injuries. This can be shown in Table 3 where majority of

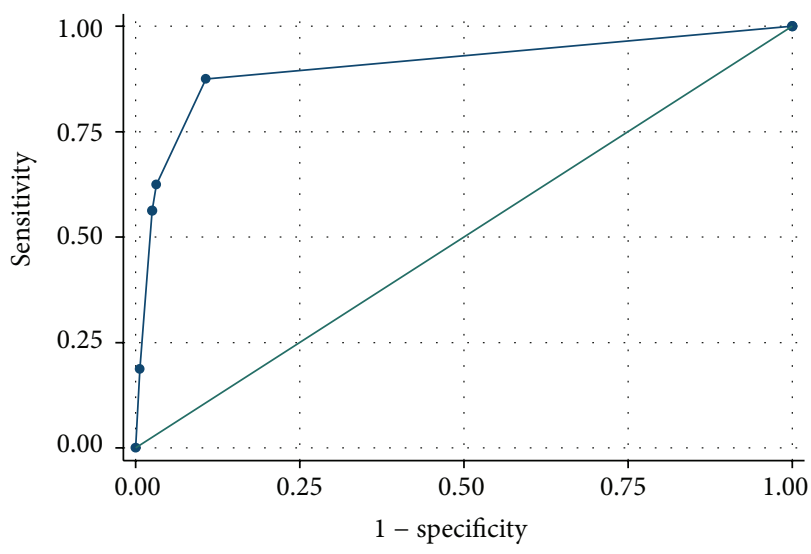

Area under ROC curve $=0.9053$

FIGURE 2: ROC curve for SSORTT score.

patient; that is; 18 patients that died; had SSORTT 0 and 23 patients had Glasgow coma score less than 8 indicating presence of severe extra-abdominal injuries.

16 patients did not have a postmortem done because the relatives declined. Haemoperitoneum was found in 3 patients and 7 patients had blunt force trauma with extra-abdominal injury. (55\%).

Majority of patients that died had a SSORTT score of 0

Majority of deaths were in the severely deranged GCS range, that is, 3-8.

Seven patients with intra-abdominal fluid died.

All patients that passed away with intra-abdominal fluid had scores of $>2$.

\section{Discussion}

We set out to determine the diagnostic accuracy of emergency ultrasound scanning using the SSORTT score in predicting the need for a therapeutic laparotomy for blunt abdominal trauma patients. The SSORTT score was defined as a sum of the ultrasound haemoperitoneum score, systolic blood pressure, and pulse rate $[19,20]$. Patients with a SSORTT score of 2 and above had a high likelihood of requiring a therapeutic laparotomy and those below that were unlikely to need one.

SSORTT scoring had excellent diagnostic accuracy for identifying patients that needed or did not need a therapeutic laparotomy with sensitivity of $91 \%$ and specificity of $90 \%$.

The findings of this study are comparable to findings by Manka et al. where patients with score of less than 1 were less likely to require a laparotomy [21].

The youths were mostly affected because they fall in the most active age bracket exposed to most injury risks $[8,22$, 23].

Motorcycle accidents accounted for most (62\%) of the injuries; the use of motorcycles a cheap means of transportation is on the increase in many countries in Africa and are a leading cause of blunt abdominal trauma [22-28]. 
TABLE 2: Laparotomy predictions at selected cutoffs.

\begin{tabular}{|c|c|c|c|c|c|c|}
\hline \multirow{2}{*}{ SSORTT score } & \multicolumn{2}{|c|}{ Standard of care } & \multirow{2}{*}{ Sensitivity } & \multirow{2}{*}{ Specificity } & \multirow{2}{*}{ PPV (\%) } & \multirow{2}{*}{ NPV (\%) } \\
\hline & Laparotomy & No laparotomy & & & & \\
\hline \multicolumn{7}{|l|}{ Score cutoffs } \\
\hline \multicolumn{7}{|l|}{ At 0} \\
\hline Laparotomy & 19 & 30 & & & & \\
\hline No laparotomy & 2 & 142 & 91 & 82 & 39 & 99 \\
\hline \multicolumn{7}{|l|}{$\geq 1$} \\
\hline Laparotomy & 19 & 17 & & & & \\
\hline No laparotomy & 2 & 155 & 90 & 90 & 53 & 99 \\
\hline \multicolumn{7}{|l|}{$\geq 2$} \\
\hline Laparotomy & 18 & 16 & & & & \\
\hline No laparotomy & 3 & 156 & 86 & 91 & 53 & 98 \\
\hline \multicolumn{7}{|l|}{$\geq 3$} \\
\hline Laparotomy & 14 & 4 & & & & \\
\hline No laparotomy & 8 & 168 & 67 & 98 & 78 & 96 \\
\hline \multicolumn{7}{|l|}{$\geq 4$} \\
\hline Laparotomy & 8 & 1 & & & & \\
\hline No laparotomy & 14 & 171 & 38 & 99 & 89 & 93 \\
\hline \multicolumn{7}{|l|}{$\geq 5$} \\
\hline Laparotomy & 5 & 0 & & & & \\
\hline No laparotomy & 17 & 172 & 24 & 100 & 100 & 91 \\
\hline \multicolumn{7}{|l|}{ At 6} \\
\hline Laparotomy & 0 & 0 & & & & \\
\hline No laparotomy & 22 & 172 & 0 & 100 & 0 & 87 \\
\hline
\end{tabular}

TABLE 3: Comparison of the fatalities and survivors.

\begin{tabular}{|c|c|c|c|c|}
\hline \multirow{3}{*}{ Injuries } & \multicolumn{2}{|c|}{ Patient status } & \multirow{3}{*}{ OR $(95 \% \mathrm{CI})$} & \multirow{3}{*}{$P$ value } \\
\hline & Alive & Died & & \\
\hline & Number (\%) & Number (\%) & & \\
\hline Bruises & $66(47)$ & $21(66)$ & $0.5(0.2-1.0)$ & 0.063 \\
\hline Abrasions & $30(21)$ & $8(25)$ & $0.8(0.3-2.0)$ & 0.661 \\
\hline Lacerations & $79(56)$ & $18(56)$ & $1.0(0.5-2.2)$ & 0.985 \\
\hline Cuts & $10(7)$ & $3(9)$ & $0.7(0.2-2.9)$ & 0.667 \\
\hline Burns & $2(1)$ & $1(3)$ & $0.5(0.1-5.1)$ & 0.519 \\
\hline Abdominal & $43(30)$ & $7(22)$ & $1.6(0.6-3.9)$ & 0.334 \\
\hline Chest & $16(11)$ & $6(19)$ & $0.6(0.2-1.6)$ & 0.262 \\
\hline Others & $120(86)$ & $23(74)$ & $2.2(0.9-5.6)$ & 0.100 \\
\hline Standard of care laparotomy & $17(11)$ & $6(18)$ & $1.6(0.6-4.9)$ & 0.370 \\
\hline \multicolumn{5}{|l|}{ SSORTT score } \\
\hline 0 & 123 & 18 & - & - \\
\hline 1 & 10 & 3 & - & - \\
\hline 2 & 2 & 0 & - & - \\
\hline 3 & 11 & 6 & - & - \\
\hline 4 & 8 & 1 & - & - \\
\hline 5 & 3 & 1 & - & - \\
\hline 6 & 2 & 4 & - & - \\
\hline \multicolumn{5}{|l|}{ Glasgow coma score } \\
\hline Severe & 13 & 21 & - & - \\
\hline Moderate & 21 & 9 & - & - \\
\hline Mild & 125 & 3 & - & - \\
\hline
\end{tabular}


Many studies have demonstrated that physical examinations are unreliable in assessing trauma patient especially those with neurological injury (brain or spinal cord), and in those with painful distracting injuries such as long bone or pelvic fractures, or in those with alcohol or other intoxicants in their systems $[29,30]$.

Eighty patients suffered head injuries (42\%) and 50 (26\%) had potentially distracting limb extremity injuries. In the absence of diagnostic aids a proportion of the severely injured would be vulnerable to delays in diagnosis of major intraabdominal visceral injuries. We contend that proper use of ultrasound in the emergency room in early identification of life threatening injuries like intra-abdominal hemorrhage, haemothorax, or haemoperitoneum may save lives in low technology settings. The additional advantage of sonographers is that it is noninvasive compared to techniques like peritoneal lavage which we many times use in the absence of sonography [22].

We found that 23 patients (12\%) received laparotomy and of these 21 were therapeutic, that is, surgically correctable lesions. Massive haemoperitoneum with solid organ injury was the commonest finding at laparotomy with ruptured spleen being the commonest solid organ injured followed by liver lacerations. There were two cases of gut perforations, one urinary bladder rupture and one with mesenteric tears.

This pattern of injuries is consistent with many other studies done in the country and the region $[6,22-26,31,32]$.

Five patients of the 23 that had laparotomy passed away, due to anesthesia related complications and aneamia or coagulopathy due to shortage of blood. The laparotomy rate in this study was $11 \%$ and the overall mortality rate was $17 \%$, comparable to previous studies $[22,23]$ which reported a mortality rate between 10 and $30 \%$.

4.1. Study Limitations. This study was not without limitations; the sensitivity and specificity of emergency US scanning could have been affected by possible sonography operator differences; however some studies show that FAST can be done reliably by both radiologist and nonradiologists $[15,33,34]$.

In the calculation of the SSORTT score, ideally blood pressure and pulse rate should be obtained at the beginning of resuscitation. However, often blood pressure and pulse rates would be taken after fluid infusions that were started to resuscitate patients; this could be assumed to influence the SSORTT score leading to an under estimation of severity of injury. Postmortem to determine cause of death were not done for various reasons like attendants declining.

\section{Conclusion}

SSORTT score is a noninvasive, reproducible test that can be performed easily and reliably predicts the need for a therapeutic laparotomy among blunt abdominal trauma patients. It should be adopted for routine use in low technology settings.

\section{Abbreviations}

AE: $\quad$ Accident and emergency

BAT: Blunt abdominal trauma

FAST: $\quad$ Focused abdominal sonography for trauma

RTC: $\quad$ Road traffic crashes

SSORTT: sum of ultrasound score, systolic blood pressure, and pulse rate.

\section{Conflict of Interests}

The authors declare that there is no conflict of interests regarding the publication of the paper.

\section{Authors' Contribution}

P. C. M. Musiitwa, M. Galukande, and R. Wangoda redesigned the experiment. P. C. M. Musiitwa collected data. M. Galukande wrote the first draft. All authors performed critical reviews for intellectual input. All authors approved the final draft.

\section{Acknowledgment}

The authors would like to thank the staff in AE unit of Mulago and Department of Surgery, College of Health Sciences, Makerere University.

\section{References}

[1] C. N. Mock, G. J. Jurkovich, D. Nii-Amon-Kotei, C. ArreolaRisa, and R. V. Maier, "Trauma mortality patterns in three nations at different economic levels: implications for global trauma system development," The Journal of Trauma, vol. 44, no. 5, pp. 804-814, 1998.

[2] J. J. Davis, I. Cohn Jr., and F. C. Nance, "Diagnosis and management of blunt abdominal trauma," Annals of Surgery, vol. 183, no. 6, pp. 672-678, 1976.

[3] W. C. Wilson, C. M. Grande, and D. B. Hoyt, Trauma: Critical Care, vol. 1, Informa Healthcare, New York, NY, USA, 1st edition, 2007.

[4] I. Matovu, Abdominal injuries in paediatrics as seen in Mulago hospital [M.S. thesis], Makerere University, Kampala, Uganda, 1978.

[5] J. A. Moylan, D. E. Detmer, J. Rose, and R. Schulz, "Evaluation of the quality of hospital care for major trauma," The Journal of Trauma, vol. 16, no. 7, pp. 517-523, 1976.

[6] D. Birabwa-Male, Abdominal injuries in Mulago hospital [M.S. thesis], Makerere University, Kampala, Uganda, 1989.

[7] W. Odero, P. Garner, and A. Zwi, "Road traffic injuries in developing countries: a comprehensive review of epidemiological studies," Tropical Medicine and International Health, vol. 2, no. 5, pp. 445-460, 1997.

[8] M. M. Ajiko, Outcome of blunt abdominal trauma in Mulago hospital [M.S. thesis], Makerere University, Kampala, Uganda, 2006.

[9] B. F. K. Odimba, "Plea for use of peritoneal lavage in management of blunt abdominal trauma victims in developing 
countries," East and Central African Journal of Surgery, vol. 3, no. 2, pp. 49-52, 1997.

[10] T. Kirschning, F. Brenner, M. Stier, C. F. Weber, and F. Walcher, "Pre-hospital emergency sonography of trauma patients," Anaesthesist, vol. 58, no. 1, pp. 51-60, 2009.

[11] K. Brohi, M. J. Cohen, M. T. Ganter, M. A. Matthay, R. C. MacKersie, and J.-F. Pittet, "Acute traumatic coagulopathy," The Journal of Trauma, vol. 54, no. 6, pp. 1127-1130, 2003.

[12] J. B. A. MacLeod, M. Lynn, M. G. McKenney, S. M. Cohn, and M. Murtha, "Early coagulopathy predicts mortality in trauma," The Journal of Trauma, vol. 55, no. 1, pp. 39-44, 2003.

[13] M. Maegele, R. Lefering, N. Yucel et al., "Early coagulopathy in multiple injury: an analysis from the German trauma registry on 8724 patients," Injury, vol. 38, no. 3, pp. 298-304, 2007.

[14] C. Tunuka, Extended focused assessment with sonography for trauma among blunt torso injury patients at Mulago hospital injury patients at Mulago hospital [M.S. thesis], Makerere University, Kampala, Uganda, 2012.

[15] G. S. Rozycki, M. G. Ochsner, J. H. Jaffin et al., "Prospective evaluation of surgeons' use of ultrasound in the evaluation of trauma patients," The Journal of Trauma, vol. 34, no. 4, pp. 516526, 1993.

[16] V. H. Thourani, B. J. Pettitt, J. A. Schmidt, W. A. Cooper, and G. S. Rozycki, "Validation of surgeon-performed emergency abdominal ultrasonography in pediatric trauma patients," Journal of Pediatric Surgery, vol. 33, no. 2, pp. 322-328, 1998.

[17] S. R. Shackford, F. B. Rogers, T. M. Osler, M. E. Trabulsy, D. W. Clauss, and D. W. Vane, "Focused abdominal sonogram for trauma: the learning curve of nonradiologist clinicians in detecting hemoperitoneum," The Journal of Trauma, vol. 46, no. 4, pp. 553-564, 1999.

[18] "Accident and emergency mulago hospital trauma registry," unpublished.

[19] O. J. Ma, M. P. Kefer, K. F. Stevison, and J. R. Mateer, “Operative versus nonoperative management of blunt abdominal trauma: role of ultrasound-measured intraperitoneal fluid levels," The American Journal of Emergency Medicine, vol. 19, no. 4, pp. 284286, 2001.

[20] K. L. McKenney, M. G. McKenney, S. M. Cohn et al., "Hemoperitoneum score helps determine need for therapeutic laparotomy," The Journal of Trauma, vol. 50, no. 4, pp. 650-656, 2001.

[21] M. Manka, R. Moscati, K. Raghavendran, and A. Priya, "Sonographic scoring for operating room triage in trauma," The Western Journal of Emergency Medicine, vol. 11, no. 2, pp. 138143, 2010.

[22] R. Wangoda, A. L. Upoki, and F. N. Owori, "Use of urethral catheters for diagnostic peritoneal lavage in blunt abdominal trauma," East and Central African Journal of Surgery, vol. 7, no. 1, pp. 63-66, 2002.

[23] O. Kobusingye, D. Guwatudde, and R. Lett, "Injury patterns in rural and urban Uganda," Injury Prevention, vol. 7, no. 1, pp. 4650, 2001.

[24] S. Bugeza, The pattern of sonographic findings in blunt abdominal trauma at Mulago hospital [M.S. thesis], Medical School Library, Makerere University, 2006.

[25] G. Ruhinda, P. Kyamanywa, D. Kitya, and F. Bajunirwe, "Abdominal injury at mbarara regional referral hospital, Uganda," East and Central African Journal of Surgery, vol. 13, no. 2, pp. 29-36, 2008.
[26] P. Chalya, J. B. Mabula, R. M. Dass et al., "Injury characteristics and outcome of road traffic crash victims at Bugando Medical Centre in Northwestern Tanzania," Journal of Trauma Management \& Outcomes, vol. 6, no. 1, atricle 1, 2012.

[27] M. Galukande, J. Jombwe, J. Fualal, and A. Gakwaya, "A bodaboda injuries a health problem and a burden of disease in Uganda: a tertiary hospital survey," East and Central African Journal of Surgery, vol. 14, no. 2, pp. 33-37, 2009.

[28] C. N. Andrews, O. C. Kobusingye, and R. Lett, "Road traffic accident injuries in Kampala," East African Medical Journal, vol. 76, no. 4, pp. 189-194, 1999.

[29] S. S. Lingawi and A. R. Buckley, "Focused abdominal US in patients with trauma," Radiology, vol. 217, no. 2, pp. 426-429, 2000.

[30] M. Blaivas, P. Sierzenski, and D. Theodoro, "Significant hemoperitoneum in blunt trauma victims with normal vital signs and clinical examination," The American Journal of Emergency Medicine, vol. 20, no. 3, pp. 218-221, 2002.

[31] S. Bugeza, E. Kiguli-Malwadde, and Z. Muyinda, "Evaluation of ultrasonography for solid organ injury in blunt abdominal trauma: experience at Mulago hospital Makerere University Uganda," in Proceedings of the 12th World Congress of the World Federation for Ultrasound in Medicine and Biology (WFUMB '09), Sydney Convention \& Exhibition Centre, Sydney, Australia, September 2009.

[32] F. Kirya, S. Kijjambu, and I. Ezati, "Outcome of major trauma at Mulago hospital in Uganda: assessment using the TRISS methodology," East and Central African Journal of Surgery, vol. 7, no. 1, pp. 59-62, 2002.

[33] G. R. Buzzas, S. J. Kern, R. S. Smith, P. B. Harrison, S. D. Helmer, and J. A. Reed, "A comparison of sonographic examinations for trauma performed by surgeons and radiologists," The Journal of Trauma, vol. 44, no. 4, pp. 604-608, 1998.

[34] S. W. Branney, E. E. Moore, S. V. Cantrill, J. M. Burch, and S. J. Terry, "Ultrasound based key clinical pathway reduces the use of hospital resources for the evaluation of blunt abdominal trauma," The Journal of Trauma, vol. 42, no. 6, pp. 1086-1090, 1997. 


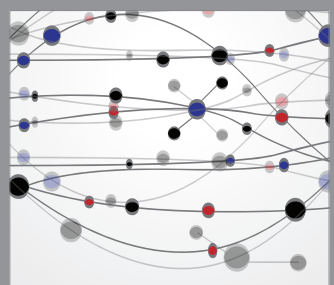

The Scientific World Journal
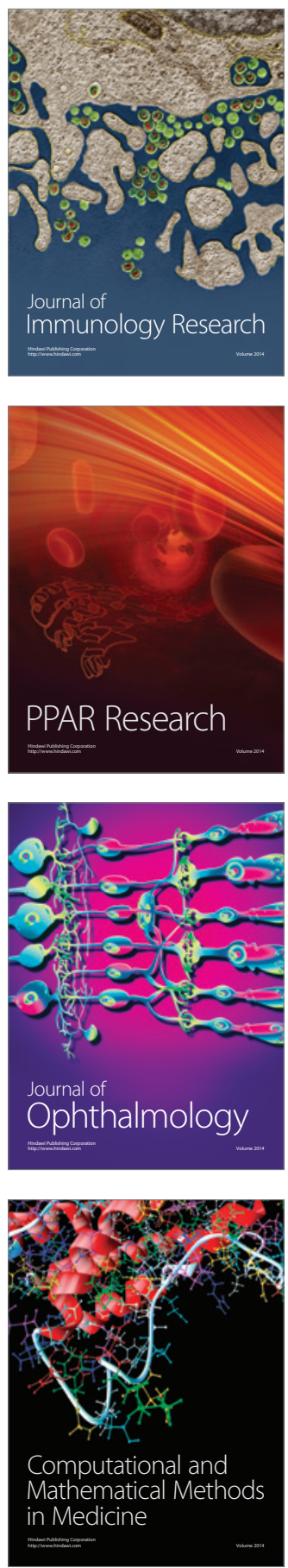

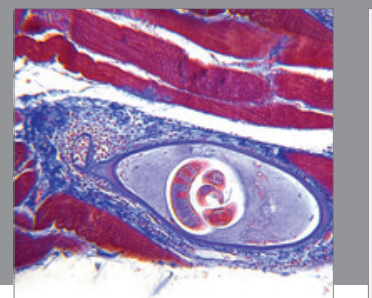

Gastroenterology

Research and Practice
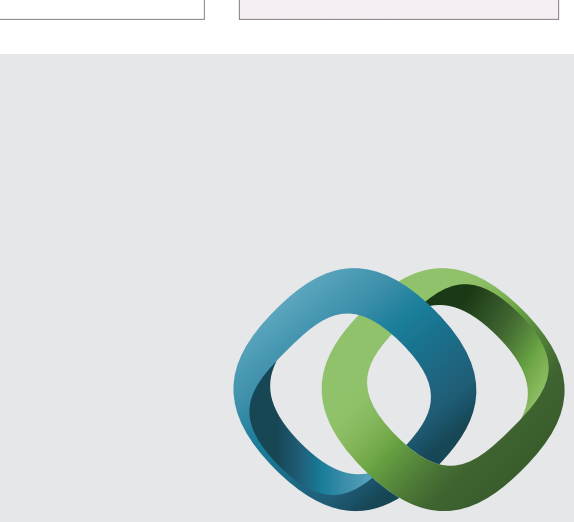

\section{Hindawi}

Submit your manuscripts at

http://www.hindawi.com
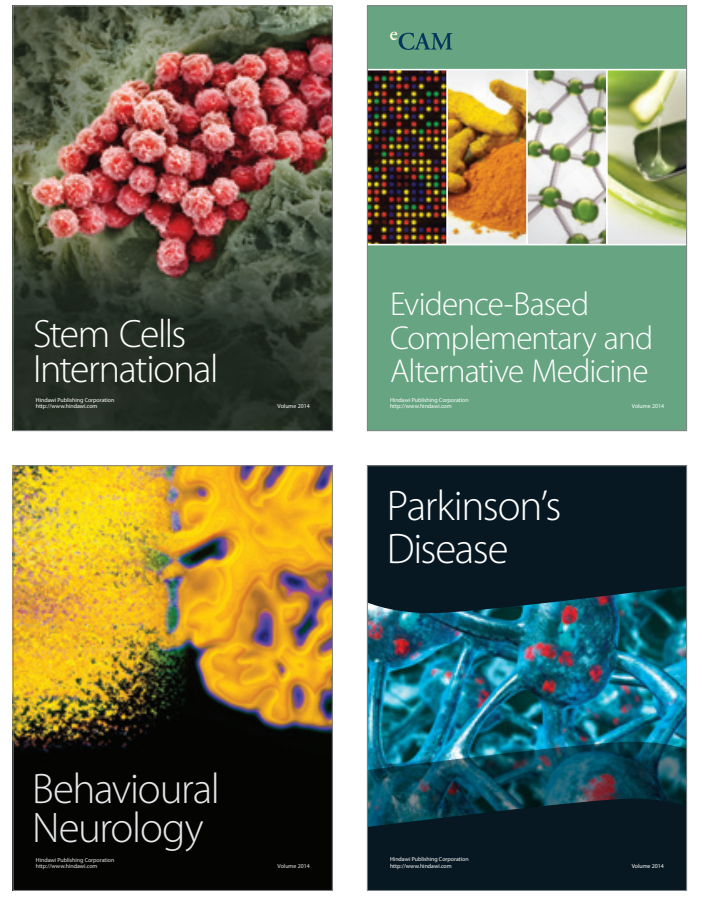
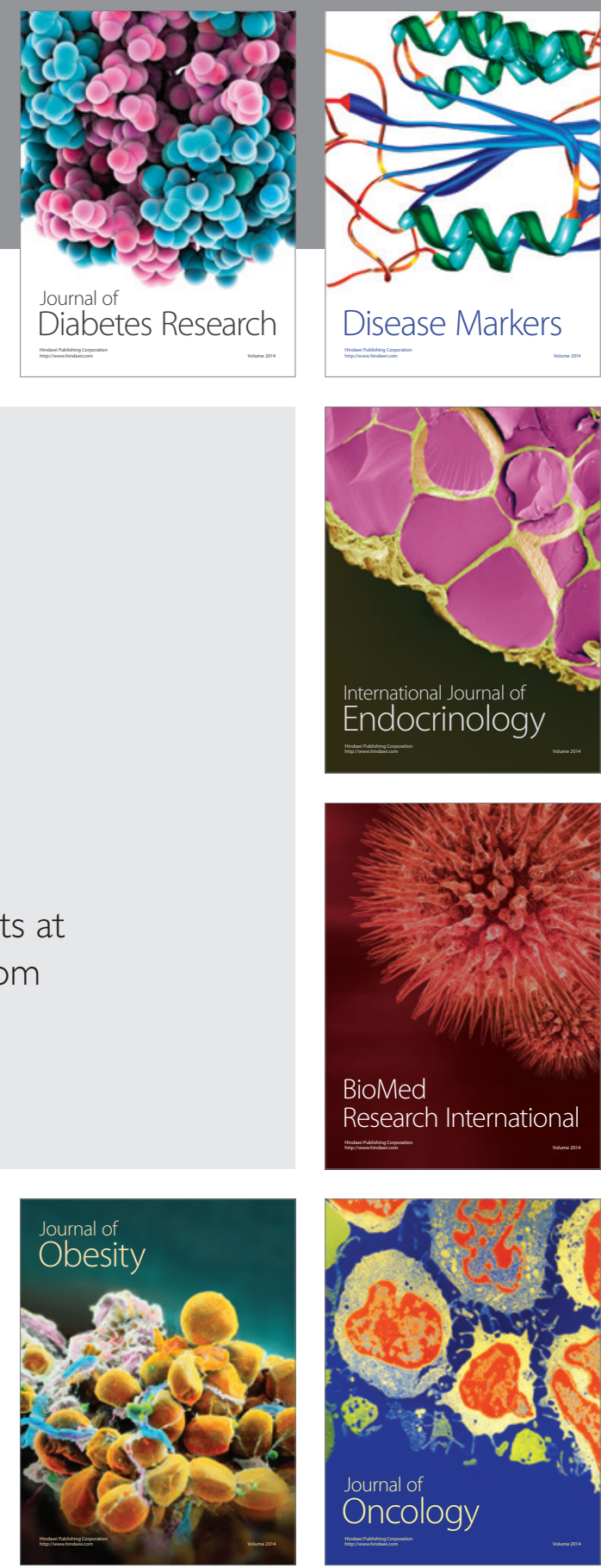

Disease Markers
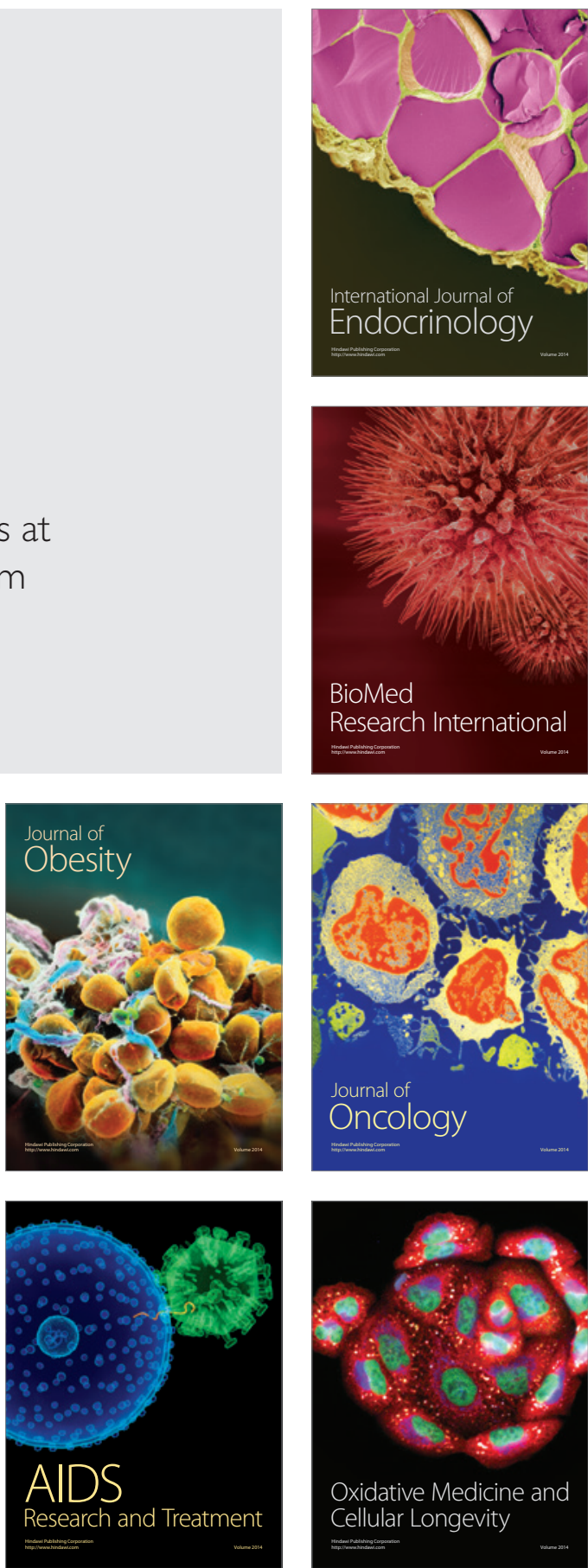